Matthieu Renault, L'Amérique de John Locke. L'expansion coloniale de la philosophie européenne

\title{
Myriam-Isabelle Ducrocq
}

\section{(2) OpenEdition}

\section{Journals}

Édition électronique

URL : https://journals.openedition.org/transatlantica/7370

DOI : $10.4000 /$ transatlantica. 7370

ISSN : 1765-2766

Éditeur

Association française d'Etudes Américaines (AFEA)

Référence électronique

Myriam-Isabelle Ducrocq, « Matthieu Renault, L'Amérique de John Locke. L'expansion coloniale de la philosophie européenne», Transatlantica [En ligne], 1 | 2015, mis en ligne le 07 janvier 2016, consulté le 31 janvier 2023. URL : http://journals.openedition.org/transatlantica/7370 ; DOI : https://doi.org/ 10.4000/transatlantica.7370

Ce document a été généré automatiquement le 31 janvier 2023.

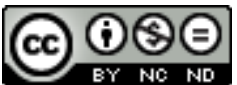

Creative Commons - Attribution - Pas d'Utilisation Commerciale - Pas de Modification 4.0 International - CC BY-NC-ND 4.0

https://creativecommons.org/licenses/by-nc-nd/4.0/ 


\title{
Matthieu Renault, L'Amérique de John Locke. L'expansion coloniale de la philosophie européenne
}

\author{
Myriam-Isabelle Ducrocq
}

\section{RÉFÉRENCE}

RENAULT, Matthieu, L'Amérique de John Locke. L'expansion coloniale de la philosophie européenne, Paris, Éditions Amsterdam, 2014, 205 p., ISBN : 978-2-35480-142-7, $16 €$

Matthieu Renault part des analyses de Carl Schmitt dans Le Nomos de la Terre pour rappeler que «l'ordre colonial impérialiste n'a jamais seulement été un ordre (géo)politique, mais aussi et inséparablement un ordre épistémique, un ordre des savoirs » (p. 12). Il faut par conséquent revenir «à la construction même de l'ordre européocentrique global, en tant qu'ordre duel, (géo)politique et épistémique, en-deçà $\mathrm{du}$ colonialisme des $\mathrm{XIX}^{\mathrm{e}}$ et $\mathrm{XX}^{\mathrm{e}}$ siècles » (p. 15), lequel a déjà fait l'objet de nombreuses études. À ce titre, la philosophie de Locke intéresse tout particulièrement l'auteur car elle «pose les fondements d'une théorie du pouvoir colonial promise à un riche avenir » (p. 26). Il s'agit néanmoins de comprendre en quoi celle-ci se distingue d' " un partage binaire-racial » qui a pu s'exprimer à la même époque et qui a légitimé l'esclavage dans les plantations. L'auteur décrit chez Locke une «conception 'continuiste' des modalités de servitude, couplée à une problématisation des différentiels de raison » qui a rendu possible " au sein d'une philosophie (inclusive) de la liberté et du consentement, la thèse (exclusive) selon laquelle il existe des êtres qui, incapables de se gouverner, doivent être gouvernés sans leur consentement » (p. 26). Il faut donc comprendre comment la pensée d'un ordre colonial repose sur «des procédés de transplantation et de transformation, de reproduction et de mutation des formes d'accumulation de la propriété et des modalités de pouvoir et de servitude d'un continent à l'autre » et d'une " logique (différentielle) du dédoublement des concepts » (p. 27). Ce sont ces procédés et cette logique que dissèque l'auteur avec une grande 
maîtrise du corpus lockien, ses textes publiés, mais aussi certains manuscrits qui se trouvent à la Bodleian et à la British Library, comme ces notes prises lors d'un voyage en France de 1676 à 1679 (qui ont fait l'objet, contrairement à ce qui est indiqué p. 93, d'une édition et d'une traduction en français récentes (cf. Carnet de voyage à Montpellier et dans le sud de la France 1676-1679, édité sous la dir. de G. Boisson, trad. de M. Rivet, Les Presses du Languedoc, 2006).

2 Pour l'auteur, l'origine de la philosophie empirique de Locke n'est pas seulement à rechercher dans le déplacement radical qu'a subi l'homme moderne, au cours de la révolution scientifique ni dans le passage "du monde clos à l'univers infini » selon la formule d'Alexandre Koyré ; elle l'est aussi dans « le décentrement de l'Ancien Monde » qui a permis de repousser les limites de la connaissance. La découverte et l'exploration de nouvelles contrées, « la nouvelle donne anthropologique » qu'elle permettait, offrait également à Locke de formidables potentialités d' "extension-expansion de l'entendement » (pp. 48-49), idée que l'auteur développe dans le premier chapitre « Le Nouveau Monde de la connaissance ». Dans le second chapitre intitulé « Géopolitique de l'état de nature ", l'auteur montre comment l'Amérique fournit à Locke la possibilité de valider sa théorie politique en démontrant la réalité, non seulement historique, mais aussi spatiale, de l'état de nature. L'auteur rappelle, un peu brièvement peut-être, que Hobbes avant Locke avait montré qu' " au sein des sociétés politiques, le retour à l'état de nature est non seulement possible, mais [qu']il est aussi très souvent réel » (p. 68). Le troisième chapitre est consacré à la question de la propriété : l'enjeu est central car il ne s'agit rien moins pour Locke que de justifier l'appropriation des terres américaines. Cette appropriation, qui procède de la même logique de maximisation agricole à l'œuvre dans le mouvement des enclosures en Angleterre, s'apparente à une véritable " expropriation sans consentement " (J. Tully). En revanche, elle entend se distinguer radicalement de la stratégie de conquête menée par les Espagnols, conquête qui ne saurait octroyer un droit de propriété à l'occupant. Comme l'écrit l'auteur la « rupture historique entre l'état de nature et l'état politique se donne à voir à présent sur la carte du monde; elle se manifeste dans l'opposition entre l'Europe et l'Amérique dont les terres sont restées telles qu'aux 'premiers âges du monde'« (p. 105). Dans le chapitre 4, l'auteur revient sur la question de l'égalité entre les individus et pose la question «Comment [Locke] pouvait-il s'accommoder de l'esclavage des plantations?» (p. 130). Pour l'auteur, l'esclavage transatlantique n'est pas un « objet » de la pensée de Locke et «reste le point aveugle d'une philosophie se définissant comme philosophie de la liberté »(p. 135). En revanche, il y a dans la théorie lockienne un processus de "métaphorisation de l'esclavage ", par lequel l'esclave ne désigne pas les victimes de la traite négrière mais, de façon plus abstraite, le sujet européen assujetti, processus d'ailleurs décrit par Mary Nyquist dans son récent ouvrage Arbitrary Rule. Slavery, Tyranny and the Power of Life and Death (2013, Chicago, University of Chicago Press). En effet, le pire degré d'asservissement pour Locke est celui de l'entendement. L'égalité chez Locke reste en fait une égalité théorique. Il existe un " différentiel de raison » qui explique une inégalité de fait, au sein des sociétés développées (ce sont les créatures oisives de l'Essai sur la Loi des pauvres qu'il faut remettre au travail), et dans les colonies à travers la figure du leet man qui apparaît dans les Constitutions fondamentales de la Caroline. Ce terme archaïque désigne « un petit blanc » qui se place volontairement sous l'entière domination de son propriétaire. Comme l'a souligné David Wootton dans son introduction aux Political Writings de Locke (Hackett Classics, 2003), ce statut héréditaire s'apparente en tous points à un système de servage qui était devenu caduc dans 
l'Angleterre du dix-septième siècle. L'auteur conclut ce chapitre en examinant quels éléments de cette philosophie ont servi à légitimer la domination des Européens sur d'autres peuples jugés plus arriérés. Enfin, dans le dernier chapitre intitulé « Variations lockiennes ", l'auteur montre comment des aspects de la théorie de la liberté et de l'égalité du philosophe anglais ont pu a contrario inspirer des thèses anticolonialistes. Il en est ainsi du Case of Ireland de William Molyneux (1698), mais aussi des «Pères fondateurs " américains qui revendiquaient son parrainage. Même si la filiation entre les idées défendues par Locke et la Révolution américaine a, depuis les années 1960, fait l'objet d'une révision, les révisionnistes - dont l'historien John Dunn - reconnaissent l'importance de la théorie lockienne de la propriété pour légitimer l'expropriation des Amérindiens « vivant dans un état de nature sans gouvernement ni loi » (Jefferson cité p. 175). L'auteur traite encore de l'usage qui a été fait de la condamnation théorique de l'esclavage par les anti-esclavagistes Bénézet et Wesley. Enfin, il examine comment le contractualisme lockien et la théorie du droit de révolte ont nourri et, pour certains critiques, perverti (c'est le point de vue d'Amartya Sen cité p. 186) la Renaissance bengalie au tournant des XVIII ${ }^{e}$ et XIX ${ }^{e}$ siècles autour des figures de Rammohan Roy et d'Ishawar Chandra Vidyasagar et la pensée politique indienne moderne incarnée par les libéraux ou par le marxiste Manabendra Nath Roy.

3 Parce que les écrits de Locke relèvent de domaines distincts et ont eu des fortunes très différentes - de l'épistémologie pour L'Essai sur l'entendement humain, de la théorie politique pour les deux Traités sur le gouvernement civil, des prescriptions législatives pour L'Essai sur la loi des pauvres, pour les Constitutions de Caroline du nord ou pour La Lettre sur la tolérance, ou bien encore de l'utopie (le passage du carnet de voyage mentionné plus haut, intitulé par Locke Atlantis) - ils ont généralement fait l'objet de traitements séparés. L'auteur les met en regard pour dresser le portrait nuancé d'un Locke, non pas seulement partie prenante de l'expansion mercantiliste et des intérêts coloniaux anglais (tout particulièrement ceux de la famille Shaftesbury), mais aussi théoricien d'un schéma de pensée qui a fourni le cadre intellectuel au colonialisme ultérieur.

4 Malgré un style vigoureux et un sens de la formule, l'auteur n'évite toutefois pas quelques affèteries de langage et fait un emploi abusif des parenthèses et des traits d'union, dont on ne voit pas toujours ce qu'ils apportent, comme dans ce passage : « Il est de nos jours de bon ton d'opposer une pensée (subversive) de la relation à une pensée (hégémonique) de la substance, comme si la seconde était la source de tous les maux (dont l'emprise des catégories raciales-racistes) et la première leur solution » ( $\mathrm{p}$. 86). Plus fondamentalement, on regrette une quasi absence des sources en français récentes, que ce soit les éditions françaises (l'édition du Carnet mentionnée plus haut ainsi que celle de l'Essai établie par P. Hamou pourtant citée p. 31 mais non recensée dans la bibliographie) ou des sources secondaires consacrées à des pans moins connus de l'œuvre de Locke (F. Lessay, Le Débat Locke-Filmer, avec la traduction du Patriarcha et du Premier Traité du Gouvernement civil, PUF, 1998, M.I. Ducrocq, De Thomas Hobbes à John Locke. Aux Sources de la démocratie anglaise, 2012). Hormis ces réserves mineures, il faut rendre hommage à l'auteur d'avoir reconstitué, pièce après pièce, l'édifice philosophique lockien, en ce qu'il était tourné vers l'Amérique et ses peuples supposés être dans un état primitif. Il montre ainsi de façon éclatante comment loin d'occuper une place marginale dans son œuvre, le continent américain tient une position tout à fait centrale en permettant de penser l'ordre colonial à partir de catégories anthropologiques (l'esprit humain, l'état de nature) ou politiques (le pouvoir, 
l'esclavage, la propriété) élaborées en grande partie à partir de l'analyse de la colonisation américaine.

\section{BIBLIOGRAPHIE}

BOISSON, Guy, Carnet de voyage à Montpellier et dans le sud de la France 1676-1679, M. Rivet, trad., Montpellier, Les Presses du Languedoc, 2006.

DUCROCQ, Myriam-Isabelle, De Thomas Hobbes à John Locke. Aux Sources de la démocratie anglaise, Paris, Presses Universitaires du Septentrion, 2012.

LESSAY, Franck, Le Débat Locke-Filmer, avec la traduction du Patriarcha et du Premier Traité du Gouvernement civil, Paris, PUF, 1998.

NYQUIST, Mary, Arbitrary Rule. Slavery, Tyranny and the Power of Life and Death, Chicago, University Press of Chicago, 2013.

WOOTTON, David, Introduction to Locke, Political Writings, London, Hackett Classics, 2003.

\section{AUTEURS}

MYRIAM-ISABELLE DUCROCQ

Université Paris 10 